\title{
Gene expression profiling of the DNMT3A R882 mutation in acute leukemia
}

\author{
XIANGNAN HUANG ${ }^{*}$, DAOXIN MA* ${ }^{*}$ WENHAO DONG, PENG LI, \\ TING LU, NA HE, TIAN TIAN, NA LIU, YAHUI DU and CHUNYAN JI \\ Department of Hematology, Qilu Hospital of Shandong University, Jinan, Shandong 250012, P.R. China
}

Received December 15, 2012; Accepted April 30, 2013

DOI: $10.3892 / \mathrm{ol} .2013 .1347$

\begin{abstract}
DNA methyltransferase 3A (DNMT3A) is one of two human de novo DNA methyltransferases essential for the regulation of gene expression. DNMT3A mutations and deletions have been previously observed in acute myeloid leukemia (AML), myelodysplastic sydromes and myeloproliferative neoplasms. However, the involvement of DNMT3A in acute lymphoblastic leukemia (ALL) has rarely been reported. In the present study, PCR and direct sequencing was performed to analyze mutations of DNMT3A amino acid residue 882 in 99 acute leukemia patients, including 57 AML patients, 41 ALL patients and a single biphenotypic acute leukemia (BAL) patient. DNMT3A expression was detected in mononuclear cells of the bone marrow in these patients and in normal individuals using real-time quantitative polymerase chain reaction, and $17.5 \%$ (10/57) of AML patients were found to exhibit DNMT3A mutations. Four missense mutations were observed in the DNMT3A-mutated AML patients, including R882 mutations and a novel single nucleotide polymorphism resulting in the M880V amino acid substitution. However, the ALL and BAL patients were not found to exhibit DNMT3A mutations. The DNMT3A expression levels in the AML patients were significantly higher compared with those of the ALL patients or normal controls. The reduced expression levels of DNMT3A were associated with a significantly lower complete remission rate in the AML patients. However, in the ALL patients, no statistical significance was identified. The results of the present study indicate that DNMT3A may play varying roles in the regulation of DNA methylation in AML and ALL.
\end{abstract}

Correspondence to: Professor Chunyan Ji, Department of Hematology, Qilu Hospital of Shandong University, 107 Wenhuaxi Road, Jinan, Shandong 250012, P.R. China E-mail: jichunyan@sdu.edu.cn

*Contributed equally

Key words: DNMT3A, R882 mutations, acute myeloid leukemia, acute lymphoblastic leukemia, gene expression

\section{Introduction}

Acute leukemia (AL) consists of a group of heterogeneous malignancies in which immature and dysfunctional hematopoietic progenitors proliferate and accumulate in the bone marrow. DNA methylation plays a key role in the pathophysiology of acute myeloid leukemia (AML) (1) and acute lymphoblastic leukemia (ALL) (2,3). DNA methyltransferase 3A (DNMT3A) is one of two human de novo DNA methyltransferases essential for regulating gene expression during cellular development and differentiation (4). DNMT3A mutations and deletions have been analyzed in AML (5), chronic myeloid leukemia (CML), chronic myelomonocytic leukemia (CMML), myelodysplastic syndrome (MDS), lymphoma and myeloproliferative neoplasms (MPNs) (6-11). The frequency of the mutations in patients with different diseases varies between $0(0 / 81$, CML patients in blast crisis) and 22.1\% (62/281, AML patients). The most common mutation has been identified at the site of amino acid residue $\mathrm{R} 882$.

DNMT3A mutations have been found to be enriched in the M4 (32.8\%) and M5 subtypes (57.1\%), according to the French-American-British (FAB) classification system (12). The DNMT3A expression levels in patients with DNMT3A mutations were observed to be marginally lower than those without mutations. In addition, DNMT3A was also found to be expressed in normal human $\mathrm{CD} 34^{+}$bone marrow cells and its expression decreased with terminal myeloid differentiation (5). However, to date, the differences in the DNMT3A expression levels in various subtypes of AML, according to FAB classification, have not been determined. In a previous study, the results of a multivariate analysis indicated that DNMT3A mutations represented independent predictive factors of poor prognosis, including a reduced overall survival (OS) and complete remission (CR) rate (7). Qiao et al (13) reported that the $\mathrm{CR}$ rate in acute leukemia (AL) patients that were identified to positively express all the DNMT genes was significantly higher than that of patients with partially positive or negative expression, indicating that DNMT3A mutations and expression may be associated with the pathogenesis and prognosis of AL.

ALL is a heterogeneous malignancy caused by the clonal proliferation of lymphocytes. The pathogenesis and causal cancer genes associated with AML and ALL differ (14). Unlike AML, extremely little is known about the mutation 
Table I. Clinical characteristics of 57 patients with AML.

\begin{tabular}{|c|c|c|c|c|c|}
\hline Characteristics & $\begin{array}{l}\text { No DNMT3A } \\
\text { mutation }\end{array}$ & $\begin{array}{l}\text { R882 } \\
\text { mutation }\end{array}$ & $\begin{array}{l}\text { Non-R882 } \\
\text { mutation }\end{array}$ & $\begin{array}{l}\text { Any DNMT3A } \\
\text { mutation }\end{array}$ & P-value ${ }^{a}$ \\
\hline Patients, $\mathrm{n}$ & 47 & 9 & 1 & 10 & \\
\hline Age at study entry, years & $42.4 \pm 16.1^{\mathrm{b}}$ & $60.3 \pm 16.5^{b}$ & 55 & $59.8 \pm 15.7^{b}$ & $0.007^{\mathrm{c}}$ \\
\hline Male gender $(\%)$ & $26(55.3)$ & $3(33.3)$ & $0(0.0)$ & $3(30.0)$ & $0.179^{\mathrm{d}}$ \\
\hline Bone marrow blasts at diagnosis, $\%$ & $77.75 \pm 20.84^{b}$ & $84.9 \pm 19.7^{b}$ & 87 & $84.9 \pm 19.7^{\mathrm{b}}$ & $0.073^{\mathrm{c}}$ \\
\hline Normal karyotype, n/total (\%) & $11 / 13(84.6)$ & 4/4 (100) & $1 / 1(100.0)$ & $5 / 5(100.0)$ & \\
\hline \multicolumn{6}{|l|}{$\begin{array}{l}\text { White-cell count at } \\
\text { diagnosis, } \times 10^{3} \text { cells } / \mathrm{mm}^{3}\end{array}$} \\
\hline Mean & $26.71 \pm 45.57^{\mathrm{b}}$ & $114.28 \pm 88.34^{\mathrm{b}}$ & 22.38 & $105.09 \pm 88.21^{\mathrm{b}}$ & $<0.001^{\mathrm{b}}$ \\
\hline Median & 10.93 & 103.00 & 22.38 & 94.47 & \\
\hline Cytogenetic risk, n/total (\%) & & & & & $0.004^{\mathrm{d}}$ \\
\hline Favorable & $18 / 45(40.0)$ & $0 / 7(0.0)$ & $0 / 1(0.0)$ & $0 / 8(0.0)$ & \\
\hline Intermediate & $19 / 45(42.2)$ & 7/7 (100.0) & $1 / 1(100.0)$ & $8 / 8(100.0)$ & \\
\hline Adverse & 8/45 (17.8) & $0 / 7(0.0)$ & $0 / 1(0.0)$ & $0 / 8(0.0)$ & \\
\hline \multicolumn{6}{|l|}{ AML subtype, n (\%) } \\
\hline M2 & $6(12.8)$ & $0(0.0)$ & $0(0.0)$ & $0(0.0)$ & \\
\hline M3 & $14(29.8)$ & $0(0.0)$ & $0(0.0)$ & $0(0.0)$ & \\
\hline M4 & $10(21.3)$ & $0(0.0)$ & $0(0.0)$ & $0(0.0)$ & \\
\hline M5 & $17(36.2)$ & $9(100.0)$ & $1(100.0)$ & $10(100.0)$ & \\
\hline
\end{tabular}

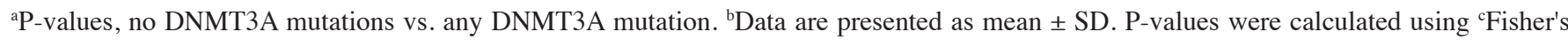
exact or ${ }^{\mathrm{d}}$ Wilcoxon tests. AML, acute myeloid leukemia.

frequency of DNMT3A in ALL patients. Therefore, in the present study, 99 Chinese AL patients were screened for DNMT3A R882 mutations, with the aim of uncovering the frequency of the R882 mutations in ALL and the relationship between ALL and AML. In addition, DNMT3A expression levels were determined in these samples and normal controls to determine whether expression levels correlate with poor prognosis. The results demonstrate that the DNMT3A mutation status in AML is an important factor to consider for risk stratification of the disease.

\section{Materials and methods}

Patients, healthy subjects and bone marrow mononuclear cell (BMMC) and peripheral blood mononuclear cell (PBMC) collection. The study recruited 99 consecutive adult patients with AL [AML, ALL and biphenotypic acute leukemia (BAL)] newly-diagnosed at Qilu Hospital of Shandong University between August 2011 and November 2012. The diagnosis was made according to the FAB classification. For the clinical analysis, CR, partial remission (PR) and non-remission (NR) were defined according to the criteria of the International Working Group (15). Cytogenetic risk was determined in the AML patients following a method described previously (16). The characteristics of the patients at the time of sampling are presented in Tables I and II. The patients with AML were treated with standard induction chemotherapy (anthracycline and cytarabine). The patients with ALL were treated with standard induction chemotherapy (vincristine, daunorubicin,
L-asparaginase and prednisone). In addition, a control group of 16 healthy donors was included. An assessment of the patient history and a physical examination were performed during the initial diagnosis. The corresponding laboratory tests were performed. BMMCs and PBMCs were obtained from $41 \mathrm{ALL}$ patients (bone marrow or whole blood), 57 AML patients, one BAL patient and 16 control individuals (bone marrow) using density-gradient centrifugation with the Ficoll-Hypaque technique (Ficoll, Pharmacia LKB Biotechnology Inc., Piscataway, NY, USA). The samples were then stored at $-80^{\circ} \mathrm{C}$.

The present study was approved by the ethics committee of Qilu Hospital, Shandong University (Jinan, China). Written informed patient consent was obtained from all participants for the treatment and cryopreservation of BM and peripheral blood according to the Declaration of Helsinki.

Genomic DNA isolation, PCR amplification and sequencing. Genomic DNA samples from bone marrow or whole blood of AML and ALL patients were extracted using the TIANamp genomic DNA kit [Tiangen Biotech (Beijing) Co., Ltd., Beijing, China] or the total DNA/RNA/protein extraction kit (Omega Bio-Tek, Inc., Norcross, GA, USA). A DNA fragment of 379 bp covering the R882 site in exon 23 of the DNMT3A gene was amplified using the S1000 thermal cycler (Bio-Rad, Hercules, CA, USA). Forward primer, 5'-TCC TGC TGT GTG GTT AGA CG-3'; and reverse primer: 5'-TAT TTC CGC CTC TGT GGT TT-3'. PCR was performed in a $25-\mu 1$ volume containing 30 ng DNA, $12.5 \mu \mathrm{l}$ PCR mastermix, $1 \mu \mathrm{l}$ forward primer, $1 \mu \mathrm{l}$ reverse primer and $\mathrm{ddH}_{2} \mathrm{O}$. The PCR conditions 
Table II. Clinical characteristics of 41 ALL patients and one BAL patient.

\begin{tabular}{|c|c|c|c|c|}
\hline Characteristics & T-cell leukemia & B-cell leukemia & $\begin{array}{l}\text { ALL with } \\
\text { unknown phenotype }\end{array}$ & BAL \\
\hline Patients, $\mathrm{n}$ & 6 & 31 & 4 & 1 \\
\hline DNMT3A mutations, n (\%) & $0(0.0)$ & $0(0.0)$ & $0(0.0)$ & $0(0.0)$ \\
\hline Age at study entry, years & $28.3 \pm 24.7^{\mathrm{a}}$ & $37.4 \pm 16.7^{\mathrm{a}}$ & $43.0 \pm 24.6^{\mathrm{a}}$ & 60 \\
\hline Male gender, n (\%) & $4(66.7)$ & $16(51.6)$ & $2(50.0)$ & $1(100.0)$ \\
\hline Bone marrow blasts at diagnosis, $\%$ & $95.0 \pm 0.0^{\mathrm{a}}$ & $86.1 \pm 13.8^{\mathrm{a}}$ & $83.5 \pm 12.0^{\mathrm{a}}$ & 97 \\
\hline Normal karyotype, n/total (\%) & 4/4 (100.0) & 9/23 (39.1) & $0 / 2(0.0)$ & $0 / 0(0.0)$ \\
\hline \multicolumn{5}{|l|}{$\begin{array}{l}\text { White cell count at } \\
\text { diagnosis, } \times 10^{3} \text { cells } / \mathrm{mm}^{3}\end{array}$} \\
\hline Mean & $52.1 \pm 67.8^{\mathrm{a}}$ & $63.0 \pm 98.4^{\mathrm{a}}$ & $2.54 \pm 1.94^{\mathrm{a}}$ & 5.08 \\
\hline Median & 16.26 & 11.17 & 2.33 & 5.08 \\
\hline \multicolumn{5}{|l|}{$\begin{array}{l}\text { Aberrant karyotype, } \mathrm{n} / \text { total }(\%) \\
(9,22)(\mathrm{q} 34 ; \mathrm{q} 11) \text { or }\end{array}$} \\
\hline BCR/ABL fusion gene & $0 / 4(0.0)$ & $13 / 23(41.9)$ & $0 / 2(0.0)$ & $0 / 0(0.0)$ \\
\hline
\end{tabular}

were as follows: denaturation at $94^{\circ} \mathrm{C}$ for $5 \mathrm{~min}$, followed by 35 cycles of denaturation at $94^{\circ} \mathrm{C}$ for $30 \mathrm{sec}$, annealing at $55^{\circ} \mathrm{C}$ for $30 \mathrm{sec}$, extension at $72^{\circ} \mathrm{C}$ for $30 \mathrm{sec}$ and ending with an extension at $72^{\circ} \mathrm{C}$ for $10 \mathrm{~min}$. The PCR products were sequenced bidirectionally using the ABI 3730xl DNA analyzer (Applied Biosystems, Bedford, MA, USA).

$R N A$ preparation and real-time quantitative PCR. The total RNA was extracted using TRIzol (Invitrogen Life Technologies, Carlsbad, CA, USA), and the cDNA was prepared using M-MLV reverse transcriptase (Promega Corporation, Madison, WI, USA) according to the manufacturer's instructions. Reverse transcription was performed at $37^{\circ} \mathrm{C}$ for $15 \mathrm{~min}$, followed by $85^{\circ} \mathrm{C}$ for $5 \mathrm{sec}$. Real-time quantitative PCR (RQ-PCR) was performed using the ABI Prism 7500 system (Applied Biosystems) according to the manufacturer's instructions. PCR was performed in a total volume of $10 \mu \mathrm{l}$, which included $5 \mu 12 X$ SYBR Green real-time PCR master mix (Toyobo Co. Ltd., Osaka, Japan), PCR-grade water, $1 \mu 1$ template cDNA and $0.5 \mu \mathrm{l}$ forward and reverse primers. The sequences of the target-specific primers were designed from human cDNA sequences available in GenBank. DNMT3A forward, 5'-GCC ACC TCT TCG CTC CGC TG-3' and reverse, 5'-GAT GAT GTC CAA CCC TTT TCG CAA-3'; and $\beta$-actin forward, 5'-TGA CGT GGA CAT CCG CAA AG-3' and reverse, 5'-CTG GAA GGT GGA CAG CGA GG-3'. The thermal cycling profile consisted of $95^{\circ} \mathrm{C}$ denaturation for $5 \mathrm{~min}$, followed by 40 cycles at $95^{\circ} \mathrm{C}$ for $15 \mathrm{sec}, 65^{\circ} \mathrm{C}$ for $15 \mathrm{sec}$ and $72^{\circ} \mathrm{C}$ for $45 \mathrm{sec}$. To exclude non-specific amplification and primer-dimer formation, a dissociation curve analysis was performed and PCR products were confirmed by agarose gel electrophoresis. PCR-grade water was used instead of template cDNA for the negative control. The fold-change in the gene expression was determined using the $2^{-\Delta \mathrm{CT}}$ method with $\beta$-actin as an endogenous control. All experiments were performed at least twice.
Statistical analysis. The Student's t-test was used to compare the differences in DNMT3A expression levels between the AML patients with R882 mutations and the normal controls. The difference in the DNMT3A expression levels between M5 subtype AML and the other AML subtypes was also compared by Student's t-test. Differences in the DNMT3A expression levels were compared by an analysis of variance in three groups. The clinical characteristics of the AML and ALL patients, including gender, age, white cell count and other factors, are presented in Tables I and II. The AML and ALL patients were categorized into high and low DNMT3A-expressing subgroups using the median value as the cut-off. Fisher's exact test was used to compare the CR rate in patients with an intermediate-risk profile. Pearson's chi-square test was used to compare the CR rates between other groups. $\mathrm{P}<0.05$ was considered to indicate a statistically significant difference. Statistical analysis was performed using the SPSS 17.0 statistical software program (SPSS Inc., Chicago, IL, USA).

\section{Results}

DNMT3A mutations. DNMT3A R882 mutational status was determined in a cohort of 57 AML and 41 ALL patients and 1 BAL patient. Of the AML patients, 6 were identified to exhibit the $\mathrm{R} 882 \mathrm{H}$ variant, two the $\mathrm{R} 882 \mathrm{C}$ variant, one the $\mathrm{R} 882 \mathrm{P}$ variant and one the M880V variant, a novel single nucleotide polymorphism that leads to amino acid substitution. The DNMT3A mutation frequency in AML was 17.5\% (10/57). Sequencing results of each type of mutation are presented in Fig. 1. None of the DNMT3A mutations were found in the ALL and BAL patients. The AML patients with DNMT3A mutations revealed lower CR rates following induction therapy compared with those with wild-type DNMT3A (0 vs. $62.8 \%$; $\mathrm{P}<0.001)$. The presence of a DNMT3A mutation was found 


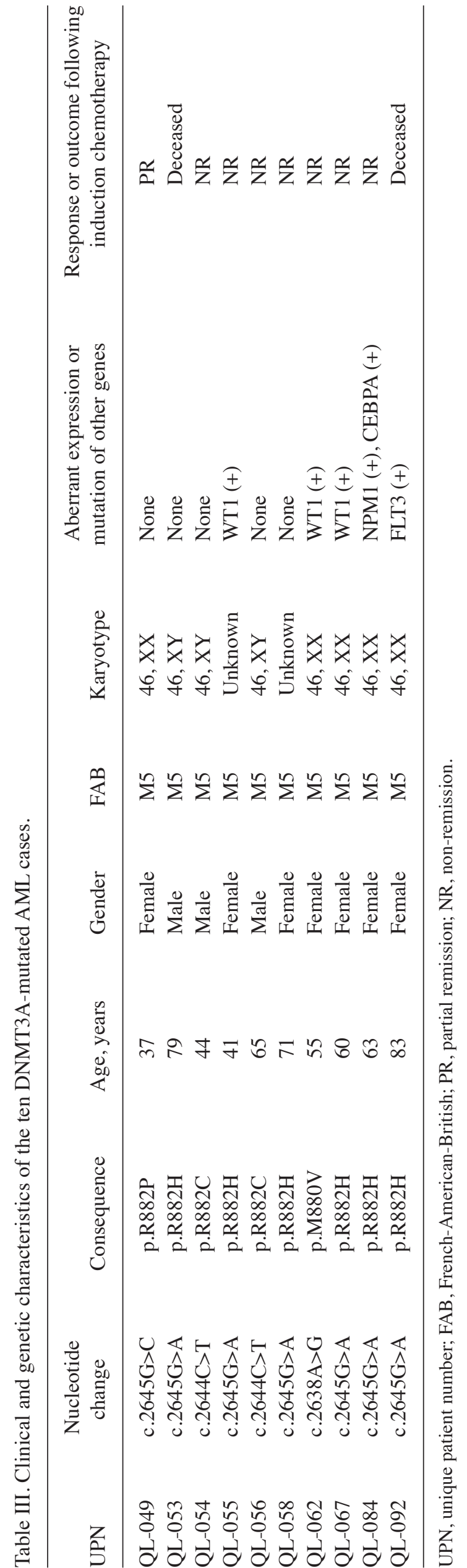

A

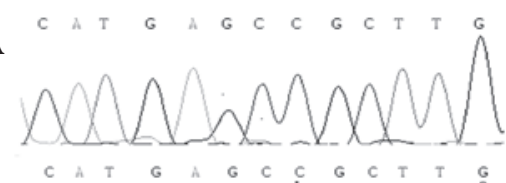

B

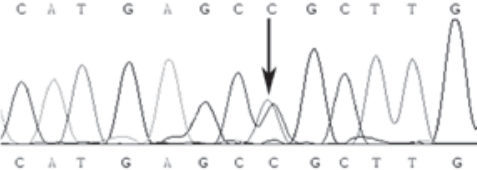

C

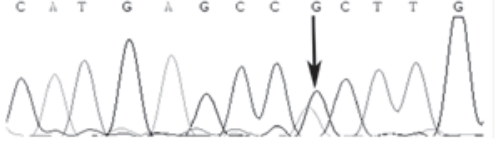

D

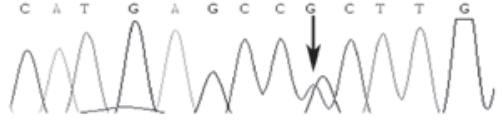

$\mathbf{E}$

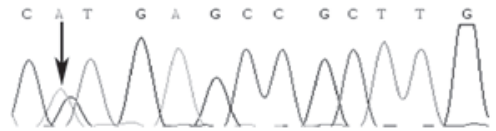

Wild type

QL -054

c. $2644 \mathrm{C}>\mathrm{T}$, p. $\mathrm{R} 882 \mathrm{C}$

QL-055

c. $2645 \mathrm{G}>$ A, p. R882H

QL-049

c. $2645 \mathrm{G}>\mathrm{C}$, p. R882P

QL-062

c. $2638 \mathrm{~A}>$ G, p. M880V

Figure 1. Sequencing of DNMT3A mutations in patients with AML. (A) Wild-type and (B-E) mutated DNMT3A gene Arrow indicates the mutated site. Three heterozygous mutations were identified at codon 882 in the samples of nine AML patients, and the sequencing figures of three patients are presented. (E) A novel SNP causing amino acid substitution, $\mathrm{M} 880 \mathrm{~V}$, was found in a female patient with AML. The unique patient number (UPN) and mutation details of these patients are provided to the right of the peak charts. DNMT3A, DNA methyltransferase 3A; AML, acute myeloid leukemia; SNP, single nucleotide polymorphism.

to correlate with a low $\mathrm{CR}$ rate in the AML patients with an intermediate-risk profile $(\mathrm{P}=0.061)$.

Clinical features of patients with DNMT3A mutations. The association between the status of DNMT3A mutations and clinical features in AML was investigated. Patients with DNMT3A mutations were classified with M5 subtype AML. The age and white cell count of the AML patients with DNMT3A mutations were higher than those without DNMT3A mutations. Gender and percentage of bone marrow blasts at diagnosis were not found to be significantly different between the two groups. DNMT3A mutations were significantly enriched in $8 / 26$ patients with a cytogenetic profile associated with intermediate risk $(30.8 \%$; $\mathrm{P}=0.004$; Table I). The clinical and genetic characteristics of the 10 DNMT3A-mutated AML cases are presented in Table III.

DNMT3A expression level. The DNMT3A expression levels were measured using RQ-PCR. DNMT3A expression in the AML patients was found to be significantly higher than that of the ALL patients or normal controls $(\mathrm{P}=0.002$ or $\mathrm{P}<0.001)$. DNMT3A expression was significantly decreased in the AML patients with DNMT3A mutations, including R882 and M880 mutations, compared with individuals without mutations $(\mathrm{P}<0.001)$. The AML patients with wild-type DNMT3A revealed significantly higher DNMT3A expression levels compared with the normal controls $(\mathrm{P}<0.001)$. No statistical difference was identified between the AML patients with DNMT3A mutations and the normal controls $(\mathrm{P}=0.747)$. The ALL patients demonstrated higher DNMT3A expression levels compared with the normal controls, however, this difference was not statistically significant $(\mathrm{P}=0.127)$. No difference was 

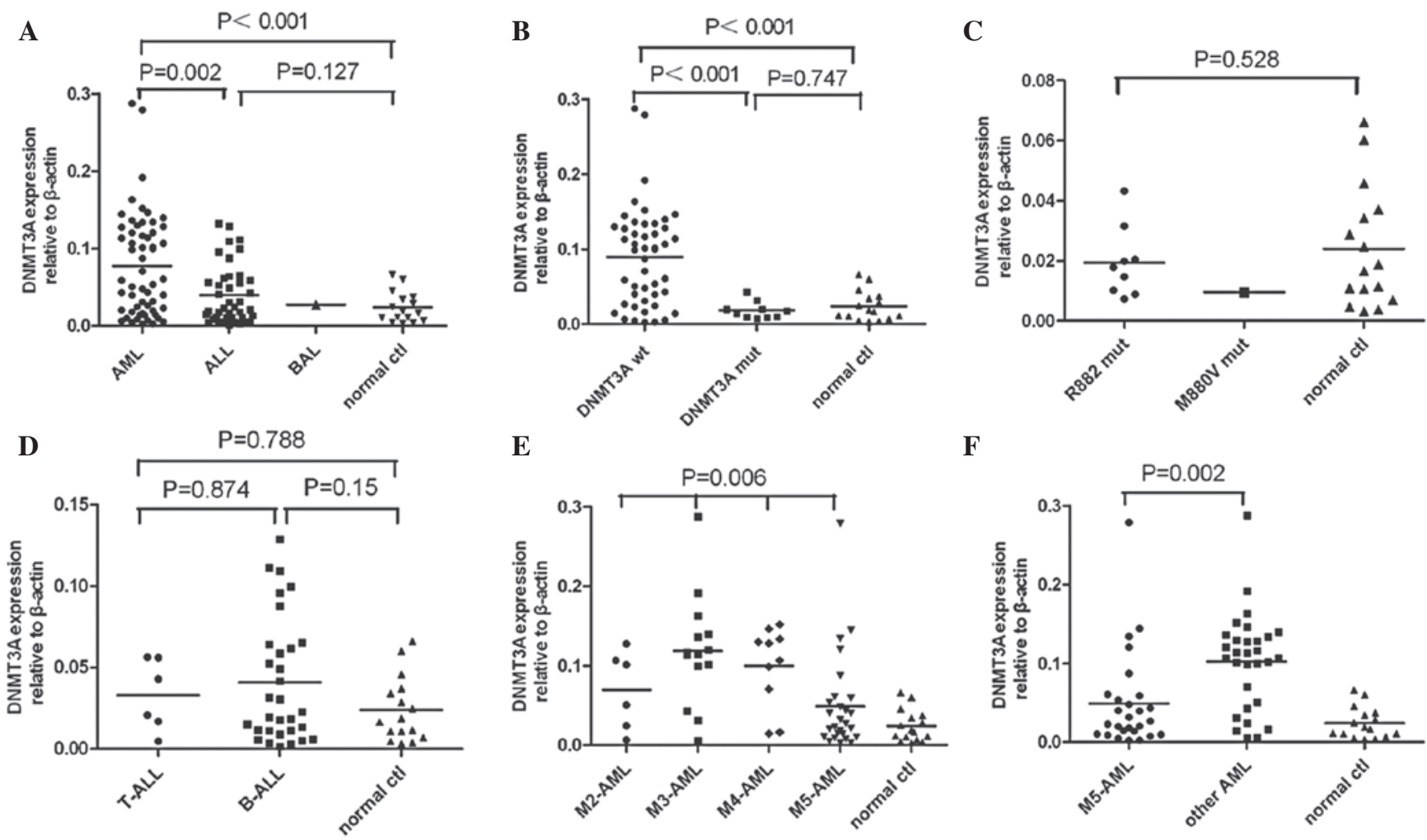

Figure 2. DNMT3A expression levels. Each dot represents one sample. (A) DNMT3A expression for 41 ALL patients, 57 AML patients, 1 BAL patient and the normal controls ( $\mathrm{n}=16$ ). DNMT3A expression in AML patients with (B) wild-type or (C) R882 or other DNMT3A mutations compared with normal controls. (D) 37 ALL patients are divided into 6 T-ALL and 31 B-ALL patients. No difference was identified between DNMT3A expression levels in T-ALL and B-ALL patients. (E) DNMT3A expression levels for various AML subtypes according to FAB classification. (F) DNMT3A expression levels for M5 subtype AML were lower compared with other types of AML. DNMT3A, DNA methyltransferase 3A; ALL, acute lymphoblastic leukemia; AML, acute myeloid leukemia; BAL, biphenotypic acute leukemia; T, T-cell; B, B-cell; FAB, French-American-British.

found in DNMT3A expression between the T-cell ALL and B-cell ALL patients $(\mathrm{P}=0.874)$. DNMT3A expression between the different AML subtypes $(\mathrm{P}=0.006)$ was significantly different. The M5 subtype AML patients were found to exhibit significantly lower DNMT3A expression levels compared with the patients with other subtypes of AML, including the M2, $\mathrm{M} 3$ and M4 subtypes ( $\mathrm{P}=0.002$; Fig. 2).

To determine whether DNMT3A expression levels affect the treatment response of AML patients, the patients were divided into 2 groups; those with low or high DNMT3A expression (below or above the median level, respectively). The CR rate was calculated for each group according to the DNMT3A expression levels. The group with low DNMT3A expression revealed a lower CR rate than that of the high DNMT3A expression group (30.8 vs. $70.4 \% ; \mathrm{P}=0.002)$. The ALL patients were also divided into two groups using the same method. No significant difference was observed in the CR rate (57.9 vs. $60 \% ; \mathrm{P}=0.894$ ) between the two groups or between T-cell and B-cell lymphoblastic leukemia (33.3 vs. $62.1 \%$; $\mathrm{P}=0.195)$.

\section{Discussion}

To date, DNMT3A mutations have been detected in AML, CML, CMML, MDS, lymphoma and MPN. The frequency of DNMT3A mutations in AML is the highest when compared with other heterogeneous malignancies. Recently, Ribeiro et al reported that mutant DNMT3A represents an independent prognostic marker in AML. When patients with DNMT3A mutations at position R882 were analyzed, an association with an inferior outcome was also observed (16). In the present study, 10 mutations were identified in DNMT3A in 10/57 (17.5\%) de novo AML patients. This high frequency is consistent with results of previous studies on DNMT3A mutations in AML patients $(5,17)$. In addition, the mutation of DNMT3A was found to correlate with a low CR rate in AML patients with an intermediate-risk profile, indicating that the mutation of DNMT3A represents a novel prognostic index for intermediate-risk AML patients. However, none of the DNMT3A R882 mutations were identified in this consecutive series of ALL cases. Prior to the present study, Kim et al reported that the frequency of DNMT3A mutations in adult ALL was extremely low $(0.8 \%, 1 / 124)(18)$. Differences in the frequency of DNMT3A mutations between AML and ALL may be associated with the different pathogenic mechanisms in AML and ALL. This hypothesis must be studied further, using larger cohorts to identify DNMT3A mutations in ALL patients and to evaluate the prognostic impact of the mutations.

In the present study, DNMT3A R882 mutations were observed to be recurrent in AML patients and associated with a poor clinical outcome. DNMT3A is markedly overexpressed in the majority of AML patients with wild-type DNMT3A when compared with normal controls. However, no difference was identified in DNMT3A expression between the AML patients with DNMT3A mutations and the control 
individuals. These results indicate that DNMT3A mutations may reduce the methyltransferase activity. Therefore, we hypothesized that the reduced expression of DNMT3A is indicative of a poor clinical outcome in AML patients due to decreased methyltransferase activity. This hypothesis is consistent with the observations that M880V and all R882 mutations are heterozygous and that this mutation reduces methyltransferase activity in vitro (19). Therefore, DNMT3A expression may represent a potential biomarker for the prediction of prognosis in AML.

In the present study, the DNMT3A expression levels in AML patients were compared between various FAB subtypes, and a significant difference was identified. DNMT3A expression in M5 subtype AML was lower than that of other AML subtypes. In addition, the frequency of DNMT3A mutations in the AML patients with the M5 subtype was higher compared with the patients of other subtypes, indicating that decreased DNMT3A expression caused by DNMT3A mutations may be associated with the incidence and progression of AML, particularly in the M5 subtype.

The difference in the CR rate between the two groups of AML patients indicated that lower DNMT3A expression correlated with an adverse treatment response. In the ALL patients, no difference was found in the CR rate, which may be explained by the marked difference in the DNMT3A mutation status and expression levels between AML and ALL. These results indicate that the function of DNMT3A in gene methylation in ALL may be distinct from its role in AML.

A number of studies have confirmed that the DNA methylation of specific genes is associated with the clinical outcome $(3,20,21)$ and that the activity of DNA methyltransferases may contribute to specific DNA methylation profiles. Previously, Challen et al analyzed the effect of hematopoietic-specific conditional Dnmt3a deletion on self-renewal in serial transplantation assays (14). Using conditional ablation, the study reported that Dnmt3a loss progressively impaired mouse hematopoietic stem cell (HSC) differentiation. Dnmt3a-null HSCs were found to exhibit increased and decreased methylation at distinct loci, including substantial $\mathrm{CpG}$ island hyper- and hypomethylation. In the Dnmt3a-null HSCs, an extremely large number of hypomethylated genes were found that are commonly overexpressed in different types of leukemia, including AML and ALL. These observations are indicative of a crucial role for Dnmt3a in the pathogenesis of malignant neoplasms. However, the Dnmt3a-null status in HSCs in mice is distinct from DNMT3A mutations in humans, as all R882 mutations are heterozygous. Whether the same set of genes is subjected to altered epigenetic patterning in DNMT3A-mutant AML cells has not been investigated.

TET2 and NPM1 mutations are markedly associated with DNMT3A mutations in T-cell lymphoma and adult AML, respectively $(5,10,17)$. These studies indicate an oncogenic cooperation between DNMT3A and other gene mutations, resulting in the deregulation of the cytosine methylation and demethylation processes. In the present study, the patients with DNMT3A mutations were older than the patients without DNMT3A mutations in AML, which was consistent with the results obtained by Ley et al (5). Consistent with these observations, a low frequency of DNMT3A mutations in pediatric
AML was observed in studies by Ho et al (0/180, 0\%) (22) and Hollink et al $(3 / 140,2.1 \%)(23)$. In pediatric AML, there is a 4-5-fold lower frequency of NPM1 mutations compared with adult AML (24). These results may partially explain why the frequency of DNMT3A mutations is low. Therefore, we hypothesized that DNMT3A mutations alone are insufficient to generate AML and other malignancies, and that second hits may be required.

The results of the present study, in combination with observations of previous studies, indicate that DNMT3A mutations are associated with adverse outcomes in AML and that they may represent a novel marker for the risk stratification of AML. By contrast, DNMT3A mutations in ALL are rare. At present, the mechanisms by which mutated DNMT3A regulates DNA methylation remain unclear. Additional studies must be performed to identify and understand the regulatory mechanisms of DNMT3A. Screening for DNMT3A mutations may provide a novel tool for the prediction of clinical outcome.

\section{Acknowledgements}

The present study was supported by grants from the National Natural Science Foundation of China (nos. 81070422, 30871088, 81070407 and 81170515), the Specialized Research Fund for the Doctoral Program of Higher Education of the Ministry of Education (no. 20100131110060) and the Independent Innovation Fund of Shandong University (IIFSDU21300072613160).

\section{References}

1. Alvarez S, Suela J, Valencia A, et al: DNA methylation profiles and their relationship with cytogenetic status in adult acute myeloid leukemia. PLoS One 5: e12197, 2010.

2. Roman-Gomez J, Jimenez-Velasco A, Agirre X, et al: Promoter hypermethylation and global hypomethylation are independent epigenetic events in lymphoid leukemogenesis with opposing effects on clinical outcome. Leukemia 20: 1445-1447, 2006.

3. Kraszewska MD, Dawidowska M, Larmonie NSD, et al: DNA methylation pattern is altered in childhood T-cell acute lymphoblastic leukemia patients as compared with normal thymic subsets: insights into $\mathrm{CpG}$ island methylator phenotype in T-ALL. Leukemia 26: 367-371, 2011.

4. Holz-Schietinger C, Matje DM, Harrison MF and Reich NO: Oligomerization of DNMT3A controls the mechanism of de novo DNA methylation. J Biol Chem 286: 41479-41488, 2011.

5. Ley TJ, Ding L, Walter MJ, et al: DNMT3A mutations in acute myeloid leukemia. N Engl J Med 363: 2424-2433, 2010.

6. Li X, Cen J, Wang Q, et al: Absence of DNMT3A gene mutation in chronic myeloid leukemia patients in blast crisis. Eur J Haematol 88: 455-457, 2012.

7. Shen Y, Zhu YM, Fan X, et al: Gene mutation patterns and their prognostic impact in a cohort of 1185 patients with acute myeloid leukemia. Blood 118: 5593-5603, 2011.

8. Jankowska AM, Makishima H, Tiu RV, et al: Mutational spectrum analysis of chronic myelomonocytic leukemia includes genes associated with epigenetic regulation: UTX, EZH2 and DNMT3A. Blood 118: 3932-3941, 2011.

9. Walter MJ, Ding L, Shen D, et al: Recurrent DNMT3A mutations in patients with myelodysplastic syndromes. Leukemia 25: 1153-1158, 2011.

10. Couronné L, Bastard C and Bernard OA: TET2 and DNMT3A mutations in human T-Cell lymphoma. N Engl J Med 366: 95-96, 2012.

11. Stegelmann F, Bullinger L, Schlenk RF, et al: DNMT3A mutations in myeloproliferative neoplasms. Leukemia 25: 1217-1219, 2011.

12. Bennett JM, Catovsky D, Daniel MT, et al: Proposals for the classification of the acute leukaemias. French-American-British (FAB) co-operative group. Br J Haematol 33: 451-458, 1976. 
13. Qiao SK, Xu SR, Guo XN and Wang Y: Clinical significance of the expression of DNA methyltransferase genes (DNMT) in acute leukemia patients. Zhongguo Shi Yan Xue Ye Xue Za Zhi 13: 260-265, 2005 (In Chinese).

14. Challen GA, Sun D, Jeong M, et al: Dnmt3a is essential for hematopoietic stem cell differentiation. Nat Genet 44: 23-31, 2011.

15. Cheson BD, Bennett JM, Kopecky KJ, et al: Revised recommendations of the International Working Group for diagnosis, standardization of response criteria, treatment outcomes, and reporting standards for therapeutic trials in acute myeloid leukemia. J Clin Oncol 21: 4642-4649, 2003.

16. Ribeiro AF, Pratcorona M, Erpelinck-Verschueren C, et al: Mutant DNMT3A: a marker of poor prognosis in acute myeloid leukemia. Blood 119: 5824-5831, 2012.

17. Yan XJ, Xu J, Gu ZH, et al: Exome sequencing identifies somatic mutations of DNA methyltransferase gene DNMT3A in acute monocytic leukemia. Nat Genet 43: 309-315, 2011.

18. Kim MS, Kim YR, Yoo NJ and Lee SH: Mutational analysis of DNMT3A gene in acute leukemias and common solid cancers. APMIS 121: 85-94, 2012
19. Yamashita Y, Yuan J, Suetake I, et al: Array-based genomic resequencing of human leukemia. Oncogene 29: 3723-3731, 2010.

20. Shen L, Kantarjian H, Guo Y, et al: DNA methylation predicts survival and response to therapy in patients with myelodysplastic syndromes. J Clin Oncol 28: 605-613, 2010.

21. Figueroa ME, Lugthart S, Li Y, et al: DNA methylation signatures identify biologically distinct subtypes in acute myeloid leukemia. Cancer Cell 17: 13-27, 2010.

22. Ho PA, Kutny MA, Alonzo TA, et al: Leukemic mutations in the methylation-associated genes DNMT3A and IDH2 are rare events in pediatric AML: a report from the Children's Oncology Group. Pediatr Blood Cancer 57: 204-209, 2011.

23. Hollink IH, Feng Q, Danen-van Oorschot AA, et al: Low frequency of DNMT3A mutations in pediatric AML and the identification of the OCI-AML3 cell line as an in vitro model. Leukemia 26: 371-373, 2011.

24. Hollink IH, Zwaan CM, Zimmermann M, et al: Favorable prognostic impact of NPM1 gene mutations in childhood acute myeloid leukemia, with emphasis on cytogenetically normal AML. Leukemia 23: 262-270, 2009. 